\title{
Sistema de calidad de servicios para negocios de alimentos y bebidas en el cantón Sucre.
}

Quality system of restaurant services for food and beverage businesses in the Sucre canton. Sistema de calidad de servicios

\section{Leyla Vanezza Jácome Villacrés ${ }^{(1)}$}

Jessika Mariela Zambrano ${ }^{(2)}$

José Ricardo Macías Barberán ${ }^{(3)}$

${ }^{1}$ Universidad Laica Eloy Alfaro de Manabí, Ecuador. e-mail: leyla.jacome@uleam.edu.ec

ORCID: https://orcid.org/0000-0002-4846-6534

${ }^{2}$ Universidad Laica Eloy Alfaro de Manabí, Ecuador. e-mail: jessica.zambrano@uleam.edu.ec

ORCID: https://orcid.org/0000-0003-1370-5934

${ }^{3}$ Universidad Laica Eloy Alfaro de Manabí, Ecuador. e-mail: jose.macias@uleam.edu.ec ORCID: https://orcid.org/0000-0002-2857-6867

Contacto: leylavjv@gmail.com

\section{Resumen}

Este trabajo es un sistema de calidad de servicio que puede ser implementado en los negocios de alimentos y bebidas y así obtener una mejora en áreas como la planificación, control y mantenimiento de los estándares establecidos por las Normas ISO 9001, específicamente orientadas a ser aplicadas en los restaurantes del cantón Sucre, donde se muestran diferentes puntos que deben ser considerados para la prestación de un servicio que busca satisfacer las necesidades de los clientes. Este sistema de gestión de la calidad es una herramienta que tiene como objetivo contribuir a la mejora de los servicios dentro del establecimiento, aprovechando el impulso que se está dando. Entre las principales técnicas empleadas se encuentran: revisión bibliográfica, observación, de carácter descriptivo e investigativo, muestreo, encuesta virtual, siendo el objeto de estudio los restaurantes de alimentos y bebidas de Sucre. 
Palabras clave: calidad, servicio, restauración, alimentos, bebidas, sistemas.

\begin{abstract}
.
This work is a service quality system that can be implemented in the food and beverage businesses and thus obtain an improvement in areas such as planning, control and maintenance of the standards established by the ISO 9001 Standards, specifically oriented to be applied in the restaurants of the Sucre canton, where different points are shown that must be considered for the provision of a service that seeks to satisfy the needs of customers. This quality management system is a tool that aims to contribute to the improvement of services within the establishment, taking advantage of the momentum that is being given. Among the main techniques used are: bibliographic review, observation, of a descriptive and investigative nature, sampling, virtual survey, the object of study being the food and beverage restaurants of Sucre.
\end{abstract}

Keywords: quality, service, restoration, food, beverages, systems.

\title{
Introducción.
}

Según (Fonseca, 2007) en su texto manifiesta que los egipcios utilizaban una amplia selección de alimentos para cocinar: guisantes, lentejas, sandía, alcachofas, lechuga, endibias, rábanos, cebollas, ajos, puerros, grasas (animales y vegetales), carne, miel, dátiles y productos lácteos, como leche, quesos y mantequilla. La historia de la restauración inicia en París, desde el año 1700 A.C. se conoce la existencia de las tabernas, así que estas eran usadas como sitios de reunión, en los inicios las tabernas solo ofrecían bebidas, vino específicamente a sus visitantes.

Las tabernas como lugares de tertulias acompañadas de bebidas ya tendrían un cambio para dar otras atenciones, eso llevaría algún tiempo en ponerse en marcha, pero con grandes satisfacciones.

En el año 1765 un hombre decide abrir una taberna y vender adicional del vino, comida, servían sopa hecha a base de patas de oveja o cerdo. Boulanger dueño de este establecimiento colocó un el letrero en la entrada de su local que decía. "Venite ad me vos qui stomacho laboratis et ego restaurabo vos" que, al castellano, podríamos traducir como: "Venid a mí todos los de estómago cansado y yo os lo restauraré", Es la última palabra usada por Boulanger (restauraré) es la que le da el nombre actual a los negocios que venden comida para deleitar el paladar de sus comensales, restaurantes. El restaurante de boulanger, Champs d'odiso, cobraba 
lo suficientemente alto como para convertirse en un lugar exclusivo en el que las damas de la sociedad acudían para mostrar su distinción. Fue el primer restaurante en Francia y en el mundo. (Melo, 2020)

Lo cual nos demuestra que desde hace ya mucho tiempo ha existido la necesidad de saciar el hambre ya sea solo por salud como por placer.

Se debe tomar en cuenta la opinión de Brillat-Savarin, un valor cultural como la cocina se convertía en universal. Para él, los cuatro requisitos de un buen restaurante era el de gozar de un ambiente distinguido, de un servicio amable y, como no, de una cocina privilegiada y una bodega sobresaliente, características de las que carecía Boulanger pero que poseía ya La Grande Taverne de Londres, regentado por Antoine Beauvilliers, antiguo jefe de cocina del Conde de Provenza, aquí sin querer refleja la calidad en el servicio completo. La palabra restaurante se estableció en breve y los chefs de más reputación que hasta entonces solo había trabajo para familias privadas abrieron también sus negocios propios o fueron contratados por un nuevo grupo pequeños empresarios: Los restauradores.

A partir de este tibio comienzo, el progreso que alcanzó el arte culinario de los restaurantes ya no se detuvo. El próximo paso fue no sólo esmerarse en la cocina sino en la manera de servir la comida al cliente. (Blogspot, 2012).

Los estándares internacionales contribuyen a hacer más simple la vida y a incrementar la efectividad de los productos y servicios que usamos diariamente. Nos ayudan a asegurar que dichos materiales, productos, procesos y servicios son los adecuados para sus propósitos.

\section{Descripción de los restaurantes.}

Los establecimientos de restauración de Sucre hoy en día se pueden encontrar con distintos diseños entre lo natural y lo moderno, en su mayoría se encuentra ubicado frente a la playa el cual brinda servicios de alimentación diaria, uso tv entre otro, por lo tanto, se dispone de otro lugar más exclusivo con servicio diferente para las personas que les gusta la privacidad y brinda hermosos paisajes. De acuerdo con los diversos lugares que se encuentra en Sucre la mayoría está ubicada en el centro con distintos diseños y gusto para cada cliente con los que brindan servicio de calidad.

\section{Localización}

Sucre, Manabí, Ecuador 


\section{Instalaciones}

Cocina, almacén, salón y servicios higiénicos

\section{Servicios que proporciona}

Servicio de cafetería

Servicio de alimentación diaria

Servicio espacio física para realizar evento.

\section{Equipamiento}

Cocina, batería de cocina, utensilios de cocina, hornos, lavavajillas, área de trabajo, neveras y congeladores.

\section{Materiales y métodos.}

La presente investigación se identifica como, descriptiva y de naturaleza investigativa, siendo el objeto de estudio, los restaurantes de alimentos y bebidas de Sucre, que permite conocer los procesos de gestión de calidad, los cuales se implementan para el mejoramiento de los mismos, en el cual se aplicó como herramienta para recolección de información encuestas vía online. Además, uno de los factores más importante de los restaurantes es que mantengan el buen manejo y el control de calidad de servicios brindados, como las normas estatales e internacionales tales como las normas ISO 900.

La investigación se la elaboró en 3 etapas; primero con un análisis foda ya que según (Sepúlveda, 2013) es una herramienta que permite conformar un cuadro de la situación actual del objeto de estudio como persona, empresa u organización, permitiendo de esta manera obtener un diagnóstico preciso que permite, en función de ello, tomar decisiones acordes con los objetivos y políticas formulados. Como segunda etapa se diseñaron encuestas virtuales dirigidas a 100 personas escogidas al azar para medir la satisfacción de los clientes en cuanto al servicio y calidad percibida por el cliente.

Tercero se interpretaron los resultados con la Puntuación de Satisfacción del Cliente (CSAT) que según (CuestioPro, 2021) indica en su artículo llamado ¿Cuál es la fórmula para medir la satisfacción del cliente?, siendo estas las opciones a escoger; Muy insatisfecho, Insatisfecho, Neutral, Satisfecho, Muy Satisfecho, como también respuestas afirmativas SI o negativas las cuales al darnos cualquiera de estas respuestas, con la siguiente formula se puede despejar la duda: 
Número de valoraciones positivas

$\operatorname{CSAT} \%=$ X100

Total de valoraciones obtenidas

Foda general de los restaurantes de Sucre.

\begin{tabular}{|c|c|}
\hline FORTALEZAS & DEBILIDADES \\
\hline $\begin{array}{l}\text { - Variedad de productos agrarios de } \\
\text { - Precios accesibles. } \\
\text { - Cocineros con conocimiento de } \\
\text { comida típica de la zona. }\end{array}$ & $\begin{array}{l}\text { - Camareros sin preparación en } \\
\text { atención al cliente. } \\
\text { - No cumplimiento de normas de } \\
\text { bioseguridad. } \\
\text { - Falta de publicidad y mercadeo. } \\
\text { - Cambios de gustos y tendencias de } \\
\text { consumo gastronómicos. } \\
\text { - Negación a actualizarse. }\end{array}$ \\
\hline OPORTUNIDADES & AMENAZAS \\
\hline $\begin{array}{l}\text { - Aumento de turistas deseosos de } \\
\text { nuevas experiencias. } \\
\text { Llegar a nuevos segmentos de } \\
\text { mercado por medio de la internet. } \\
\text { - Revolución tecnológica }\end{array}$ & $\begin{array}{l}\text { - Rivalidad de los competidores. } \\
\text { - Apertura de nuevos restaurantes. } \\
\text { - Falta de actualización y tendencias } \\
\text { gastronómicas. } \\
\text { - Fenómenos naturales. }\end{array}$ \\
\hline
\end{tabular}

Tabla 1. Foda.

\section{Encuestas}

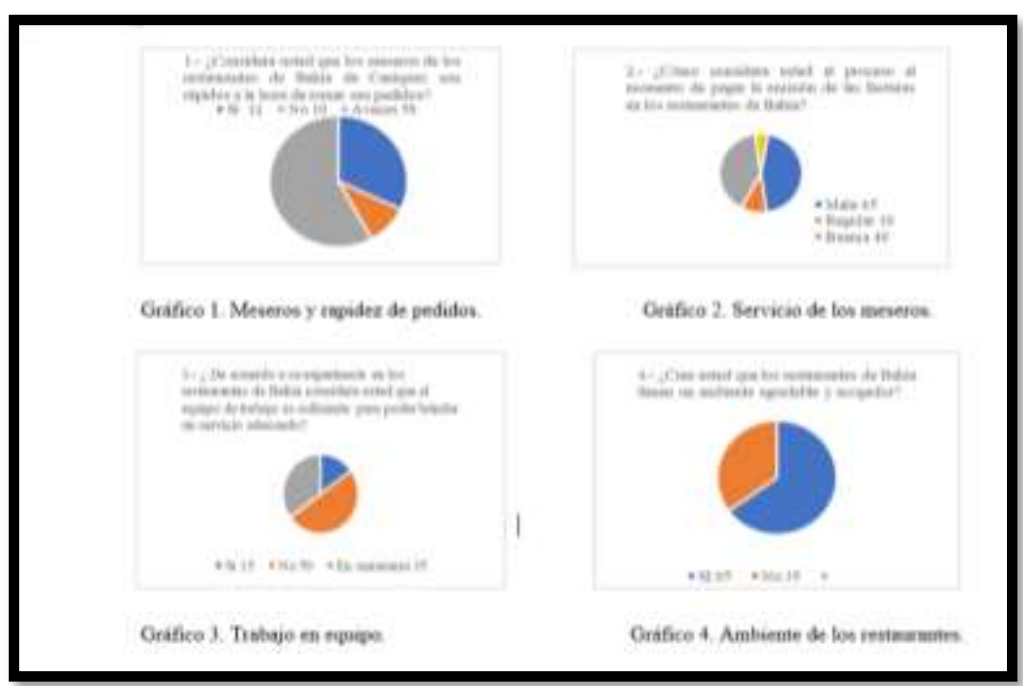




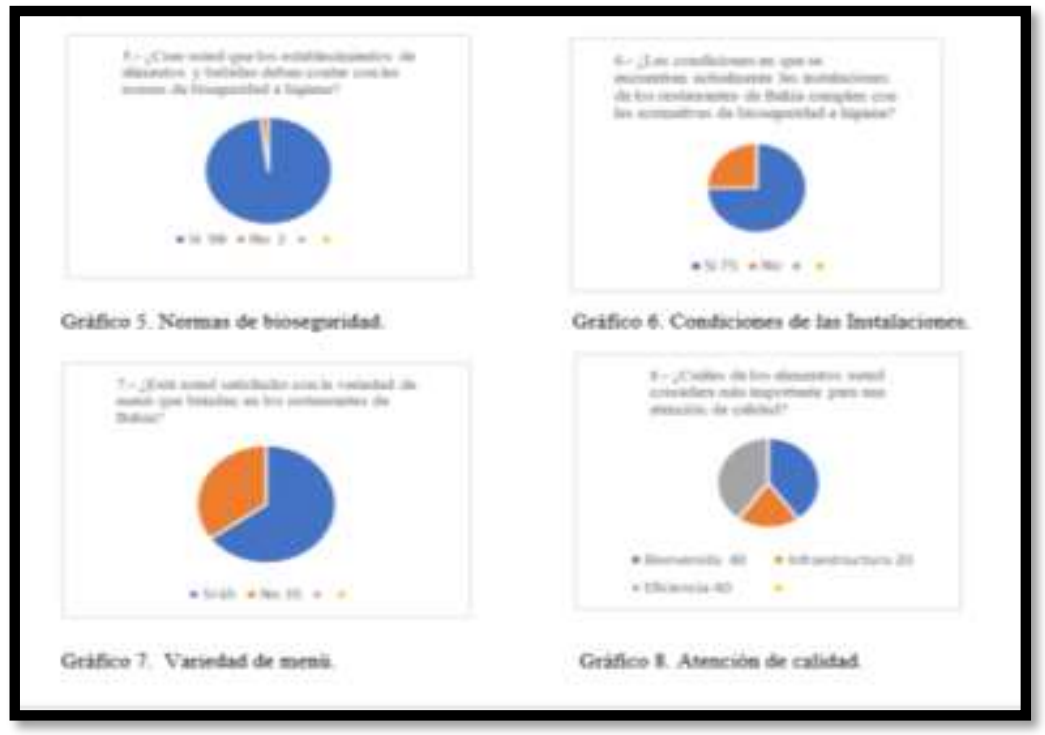

\section{Resultados.}

En las encuestas realizadas se pudo resumir lo siguiente:

\begin{tabular}{|c|c|c|c|}
\hline Preguntan & $\begin{array}{l}\text { H de } \\
\text { valoraciones } \\
\text { positivas. }\end{array}$ & $\begin{array}{l}\text { Total de } \\
\text { valoracionen } \\
\text { obtenidas. }\end{array}$ & CSAT $\%$ \\
\hline $\begin{array}{l}\text { 1.- LConsidera usted que los meseros de los } \\
\text { restaurantes de Bahia de Caránuez son rapidos a la } \\
\text { hora de tomar sus pedidos? }\end{array}$ & 32 & 100 & $32 \%$ \\
\hline $\begin{array}{l}\text { 2.- ¿Como conaidera usted el proeeso al momento de } \\
\text { pagar la emision de las faeturas en los restaurantes de } \\
\text { Bahia? }\end{array}$ & 40 & 100 & $40 \%$ \\
\hline 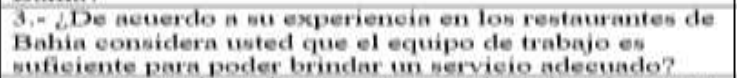 & 15 & 100 & $15 \%$ \\
\hline $\begin{array}{l}\text { 4.- LCree usted que fon restaurmites de Bahin tienen un } \\
\text { ambiente agradable y aeogedor? }\end{array}$ & 65 & 100 & $65 \%$ \\
\hline $\begin{array}{l}\text { 5. ¿Cree ubted que los establecimientos de alimentos y } \\
\text { bebidas deben eontar eon tas noman de bioneguridad a } \\
\text { higiene? }\end{array}$ & 98 & 100 & $98 \%$ \\
\hline $\begin{array}{l}\text { O.- Lan condioiones en que ve eneventran actualmente } \\
\text { las instalooiones de lon restaurantea de Bahia eumplen } \\
\text { con las normativas de bioseguridad e higiene? }\end{array}$ & 75 & 100 & $75 \%$ \\
\hline $\begin{array}{l}\text { 7.- LEata usted satistecho eon la variedad de meno que } \\
\text { brindan en los restaurantes de Bubia? }\end{array}$ & 65 & 100 & $65 \%$ \\
\hline
\end{tabular}

\section{Tabla 2. Resultado de la Puntuación de Satisfacción del Cliente.}

En la primera pregunta realizadas a las cien personas respondieron que solo en el $32 \%$ de los casos existe rapidez en el servicio a la hora de tomar el pedido en los restaurantes, dejando un amplio 68\% de insatisfacción en esta opción. 
En la segunda pregunta en cuanto al proceso al momento de cancelar la cuenta, el $40 \%$ respondieron que se sienten satisfechos dando por negativo al $60 \%$ que consideró mala atención al respecto.

En la tercera pregunta los clientes manifestaron en un $15 \%$ que el equipo de trabajo es suficiente para poder brindar un servicio adecuado, lo cual deja a un $85 \%$ de clientes insatisfechos o con una observación negativa.

En la cuarta pregunta respecto al ambiente del lugar el $65 \%$ respondieron positivamente que, si tienen un ambiente agradable y acogedor, el 35\% respondieron que no tiene un ambiente agradable, esperando siempre algo más quizá tienen la necesidad de renovar o innovar.

En la quinta pregunta respecto a la bioseguridad el $98 \%$ de los encuestados respondieron que los establecimientos de alimentos y bebidas deben contar con las nomas de bioseguridad e higiene, solo un $2 \%$ respondió que no por el desconocimiento de la importancia de las normas de bioseguridad e higiene.

En la pregunta número seis los encuestados respondieron en un $75 \%$ que a pesar de las condiciones en que se encuentran actualmente las instalaciones de los restaurantes de Bahía si cumplen con las normativas de bioseguridad e higiene, un $25 \%$ respondieron que no cumplen con estas normativas de suma importancia.

En la séptima pregunta en cuanto al menú, el 65\% respondió si estar satisfecho con la variedad de menú que brindan en los restaurantes de Bahía, dejando al 35\% insatisfecho.

En la pregunta número ocho donde los clientes escogen cuál de los elementos consideran más importante para una atención de calidad, el $40 \%$ escogió la bienvenida, otro $40 \%$ la eficiencia, dejando en último lugar a la infraestructura con un $20 \%$.

\section{Discusión.}

\section{Sistema de calidad ISO.}

Garantizar la calidad y salubridad de los alimentos que un establecimiento ofrece a sus clientes, debería ser una prioridad. Es indispensable implantar un sistema de gestión alimentaria, que ayude a controlar el alimento desde su compra y almacenamiento hasta el consumo. Para ello es imprescindible que esté implicado y concienciado todo el personal con la NORMA ISO 22001 de seguridad alimentaria, la cual proporciona las reglas a seguir. Además, ofrece un sello oficial de calidad que certifica que el establecimiento cumple con la norma. La "calidad turística" es otra normativa de calidad, que de tenerla implantada proporciona a los establecimientos prestigio y una mejor imagen delante de sus clientes. ${ }^{i}(\mathrm{ISO}, \mathrm{s} / \mathrm{f})$ 
Existen una amplia gama de sistemas de gestión de calidad, que son muy apropiados para implantar en un restaurante o bar. Los establecimientos que dispongan de alguno de ellos, sin duda gestionarán mejor sus recursos, optimizarán sus procesos y serán más competitivos.

\section{ISO 9001 (Gestión de la calidad).}

Esta norma consigue estandarizar la gestión de los procesos sobre los servicios que se ofrecen, consiguiendo un mayor control sobre ellos. Permite implantar el círculo de la mejora continua dentro de la empresa y a cumplir con la legislación vigente. (TURISTICAS, 2020)

Para los restaurantes, garantizar la salubridad de los alimentos que se van a consumir debe ser una prioridad. Por ello se ha encontrado un sistema con el que se asegura su correcta gestión, desde su adquisición inicial hasta el consumidor final. Permite implantar mejoras de forma continua dentro de los mismos sin dejar de cumplir la legislación vigente.

\section{ISO 22001 (Gestión alimentaria).}

La norma 22001 ayuda a asegurar la calidad de productos y servicios en toda la cadena de suministro, cuidando principalmente las condiciones de elaboración para asegurar la protección del consumidor entregando productos y servicios confiables, demostrando el compromiso por la mejora continua. (Qualite, 2019)

Para las empresas, y especialmente las turísticas, es altamente recomendable poder implantar sistemas basados en APPCC (Análisis de Puntos Críticos). El APPCC es un sistema de control que garantiza la vigilancia de los peligros de tipo microbiológico, físico o químico. .

\section{Beneficios de contar con un Sistema de Gestión en Inocuidad Alimentaria:}

- Control de riesgos ampliado: Con el fin de mejorar la manera en que se solucionan los problemas dentro de la cadena de suministro, todo esto con el fin de evitar todo tipo de fallos y a disminuir los costos.

- Mejor cumplimiento de la legislación aplicable: Esto permite hacer verificaciones constantes para comprobar que se cumplieron todos los requisitos legales.

- Evitar fraudes alimentarios: Esta versión de la norma que nos permite evitar fraudes alimentarios que puedan poner en riesgo la salud a través de análisis de vulnerabilidades. 
- Optimización de recursos: Debido a los rigurosos procesos exigidos, la norma está diseñada para que uses mejor los recursos disponibles, lo cual se traducen un ahorro substancial en costos, tanto internamente como en toda la cadena de producción.

- Integración con otras normas: Esta norma está diseñada para facilitar la implementación con otros sistemas de gestión (como ISO 9001 ó 14001) con el fin de facilitar a la empresa contra con las ventajas de disponer de varias normas sin aumentos innecesarios de la documentación.

\section{Calidad en el servicio de restaurantes.}

La calidad significa aportar valor al cliente, y esto no es más que ofrecer unas condiciones de uso del producto o servicio superiores a las que el cliente espera recibir y a un precio accesible. Algunos expertos, definen calidad como: "Entregar al cliente no lo que quiere, sino lo que nunca se había imaginado que quería y que una vez que lo obtenga, se dé cuenta que era lo que siempre había querido".

Para que un restaurante logre la calidad en su servicio, este debe cumplir con las necesidades y expectativas de sus clientes, ya que ellos son lo más importante y quienes determinan que tan bueno es el servicio ofrecido por un establecimiento en particular. (Sanchez, Avalos, \& Vinueza, 2019)

Para conocer cuáles son las necesidades y expectativas de los clientes, y así poder ofrecer un servicio de alta calidad en un restaurante, es necesario medir las percepciones de los clientes con respecto a conceptos específicos que constituyan el servicio en general.

Deben tener en cuenta los propietarios y gerentes de la restauración, es que manejan una empresa que forma parte de la industria alimentaria y como tal, es importante adherirse a las reglas básicas que garanticen la inocuidad de sus clientes. garantiza una experiencia memorable que motive próximas visitas, así como la influencia sobre otros comensales que repitan una y otra vez el ciclo de recompra, que garantiza la rentabilidad del negocio.

\section{Costos de calidad.}

Se denomina costos de la calidad lo que le cuesta a la organización desarrollar la función de la calidad, es decir, lo que gasta produciendo con calidad (evitando, previniendo o detectando los errores, inspeccionando los procesos, etc.), y también lo que cuestan los errores producidos. Aunque se ha intentado muchas veces unificar 
los conceptos existentes sobre el coste de la calidad, todavía no se ha conseguido definir los elementos que la componen de manera unívoca. (Riquelme, 2017)

Los costos de la calidad forman parte de una importante inversión, que cualquier empresa debe tener y hacer, para ir en busca del crecimiento progresivo de la productividad, la calidad y la rentabilidad debe de ser prioridad dentro de un establecimiento de restauración.

\section{Costos de prevención.}

Los costos de prevención se obtienen a partir de la suma del coste de todas las actividades que tienden específicamente a evitar una calidad deficiente de servicios. Es decir, son los que se producen cuando se intentan reducir o evitar los errores. (Serrano, 2017)

Son aquellos costos destinados para prevenir y evitar errores, fallas y desviaciones durante todo el proceso productivo, que desmeriten la calidad del producto. Con una alta inversión en estos, se espera que disminuyan los costos de fallas.

\section{Costos de evaluación.}

Los costos de evaluación están relacionados con la medición, evaluación o auditoría de servicios para asegurar que se adaptan a las normas de calidad y a los requisitos de comportamiento establecido. Es decir, son el total de gastos originados para intentar determinar si una actividad se ha realizado correctamente. (Serrano, 2017)

\section{Clasificación de los indicadores que miden los costos en el restaurante:}




\begin{tabular}{|l|l|l|l|l|}
\hline Indicadores & Eficacia & Eficiencia & Proceso & Resultado \\
\hline Ingresos & & $\mathrm{x}$ & & $\mathrm{x}$ \\
\hline Ventas & & $\mathrm{x}$ & & $\mathrm{x}$ \\
\hline Costos gastronómicos & & $\mathrm{x}$ & & $\mathrm{x}$ \\
\hline Gastos operacionales & & $\mathrm{x}$ & & $\mathrm{x}$ \\
\hline Otros gastos. & & $\mathrm{x}$ & $\mathrm{x}$ & $\mathrm{x}$ \\
\hline Gastos de combustibles, energía, agua. & & $\mathrm{x}$ & & $\mathrm{x}$ \\
\hline Salarios y seguridad social. & & $\mathrm{x}$ & $\mathrm{x}$ & $\mathrm{x}$ \\
\hline Cantidad de clientes & & $\mathrm{x}$ & & $\mathrm{x}$ \\
\hline Per cápita (promedio) & & $\mathrm{x}$ & $\mathrm{x}$ \\
\hline Aprovechamiento de la capacidad instalada \\
(promedio).
\end{tabular}

Tabla 3: Indicadores que miden los costos en el restaurante.

\section{Certificaciones de calidad aplicables en restauración.}

Los líderes de la calidad y muchos estudios realizados empíricamente han señalado. Que la gestión de la calidad es un elemento clave para mejorar los resultados de la empresa. Una forma de implantarla es a través de la norma ISO 9001 y numerosos estudios han analizado el proceso de implantación y certificación de la norma, así como sus beneficios. 
- No obstante se define la certificación de calidad a los resultados de un proceso que implica la satisfacción de todas las necesidades, exigencias y expectativas legítimas de los consumidores, respecto a los productos y servicios, a un precio aceptable, de conformidad las condiciones contractuales mutuamente aceptadas y con los factores subyacentes que determinen la calidad tales como la seguridad, la higiene, la accesibilidad, la transparencia, la autenticidad y la armonía de una actividad de restaurante y bebidas que preocupada por su entorno humano y natural.

- El valor de calidad como medio de discriminación de precios y fuente de ventajas competitivas que se apoye con el reconocimiento en el mercado.

- Implantación interna de acciones de cambios organizativos para mejorar el desempeño que sean consecuente con ella.

- Los certificados y acreditaciones de calidad deben de facilitar el objetivo a través de la estandarización.

- Diferenciación basada en la calidad como estrategia competitiva frente a rivales emergentes como ventajas comparativas en precios.

- Tener una buena calidad en las instalaciones como a los sistemas de gestión.

Sistema de calidad de servicio de restauración para negocios de alimentos y bebidas.

Organigrama del restaurante.

El organigrama de un restaurante es la representación gráfica de la estructura que comprende cada uno de los empleados que se encuentran laborando dentro de la organización, junto a sus dueños y gerentes, resaltando las relaciones jerárquicas y las competencias que le dan vida al mismo. (Riquelme, 2019).

La gestión interna de un restaurante juega un papel clave para el éxito o el fracaso del mismo. El organigrama de un restaurante debe responder a las necesidades del mismo para mejorar la comunicación entre los diferentes empleados y, con ello, hacer un mejor seguimiento de las actividades que tienen que llevar a cabo cada uno de ellos.

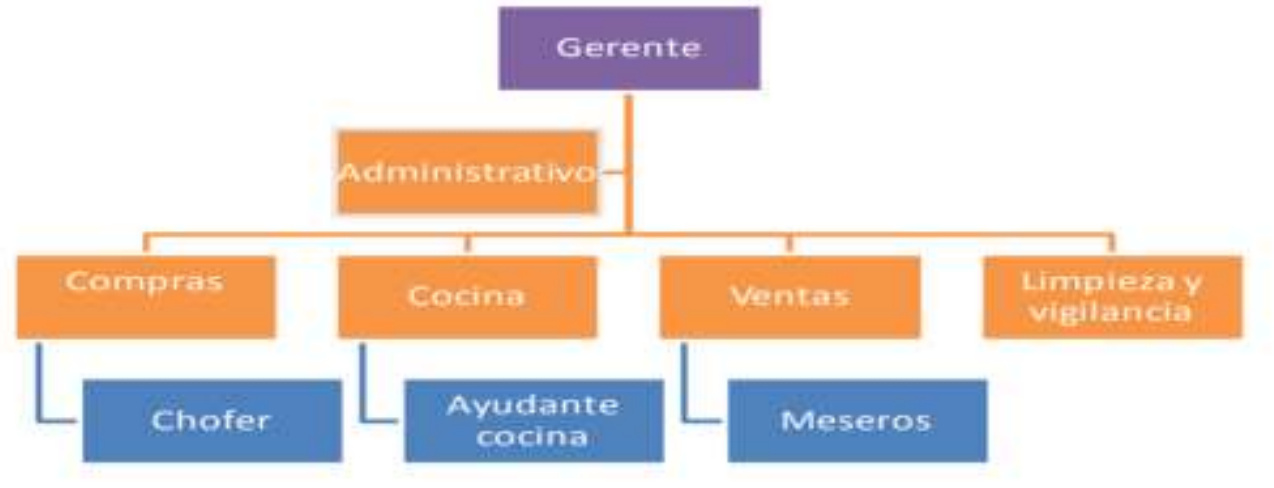


Gráfico 9. Organigrama de un negocio de restauración.

\section{Política de calidad y objetivos de calidad.}

La política de la calidad debe:

a) Estar disponible y mantenerse como información documentada.

b) Comunicarse, entenderse y aplicarse dentro de la organización.

c) Estar disponible para las partes interesadas pertinentes, según incumba.

La política de calidad es un documento que se encuentra respaldado por la dirección. En la norma ISO 9001:2015 se divide este apartado en dos:

En el primero se describen todos los requisitos que cumplen política de la calidad, además se adecuarse a la empresa.

En el segundo se deben incluir todas las obligaciones que se deben cumplir, como puede ser la disponibilidad de los productos para las partes interesadas.

Se puede destacar que es un documento que se debe aplicar y adecuar al contexto de la empresa, no solamente al propósito de ésta.

La organización debe conservar información documentada sobre los objetivos de la calidad. Los objetivos de calidad tienen que ser coherentes con la política de calidad de la empresa y ser notorio para establecer la conformidad de los productos y los servicios, además de la mejora en la satisfacción del cliente. Los objetivos de la calidad tienen que medirse, contar con el cliente y los requisitos legales deben estar vigilados para determinar si se cumplen o no. Se deben realizar comunicaciones cada cierto tiempo que tienen que estar actualizadas.

\section{Objetivos de la calidad}

\begin{tabular}{|l|ll|}
\hline $\begin{array}{l}\text { Ser acertados para la conformidad } \\
\text { de los productos y servicios y para } \\
\text { el aumento de la satisfacción del } \\
\text { cliente. }\end{array}$ & - $\begin{array}{l}\text { Atender y responder las solicitudes } \\
\text { de los clientes. }\end{array}$ \\
\hline $\begin{array}{l}\text { Ofrecer una atención al cliente y } \\
\text { un servicio amable y } \\
\text { personalizado. }\end{array}$ & - $\begin{array}{l}\text { Dar información clara y eficaz a } \\
\text { cada uno de nuestros clientes. }\end{array}$ \\
\hline
\end{tabular}

Gráfico 10: Objetivos de calidad.

\section{Requisitos del cliente, requisitos implícitos del restaurante y requisitos legales}

Es necesario utilizar la emotividad, es decir, usar los sentimientos con el cliente para de este modo ganar la confianza del mismo dando como resultado una mejor relación. 
- En primer lugar, para poder determinar e implantar planes para realizar todos los procesos debe ser primordial para asegurar el cumplimiento de los requerimientos de los clientes internos y externos.

- Fomentar procedimientos de control, verificación y validez, del diseño y desarrollo del producto de tal manera que se obtenga seguridad al cumplir con todos los requisitos especificados por el cliente, organismo de gobierno y estatutos de ley. (OCAÑA, 2018).

\section{Mapa de procesos del restaurante}

El mapa de procesos es la representación gráfica, o sea el diagrama, de la interrelación existente entre todos los procesos y subprocesos de la empresa. Dicho así, puede parecer fácil de realizar, pero la verdad es que suele ser una tarea ardua que implica a los diferentes departamentos de la empresa. (MESTRES, 2019).

El mapa de procesos recoge la interrelación de todos los procesos que realiza una organización. Un proceso es el conjunto de actividades y recursos interrelacionados que transforman los elementos de entrada en elementos de salida aportando valor para el usuario.

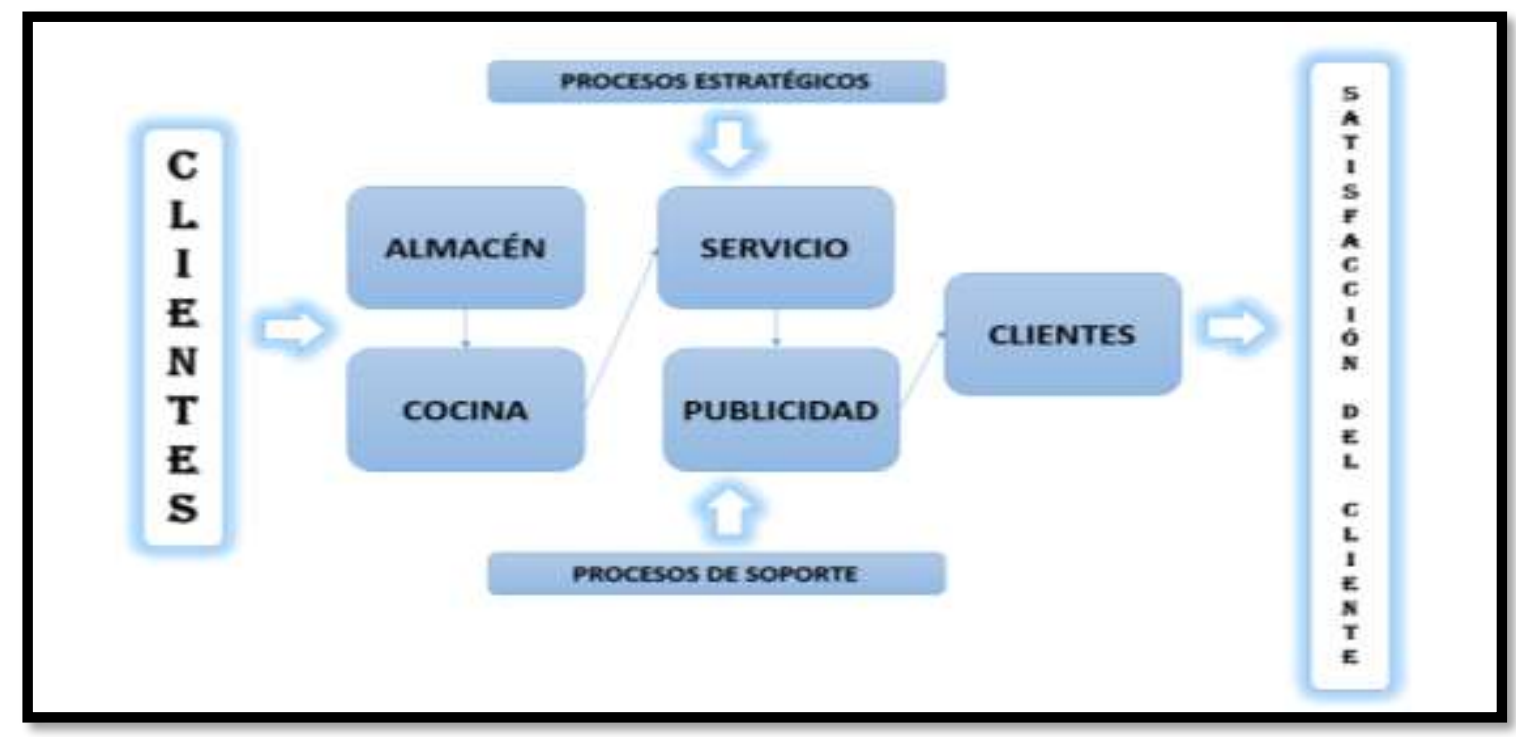

Gráfico 11. Mapa de Proceso.

\section{MAPA DE CADA PROCESO}

Una vez determinados los procesos de un restaurante, se desarrolla un mapa de cada proceso en el cual se va a implementar el sistema de calidad, en este se anota el propósito del mismo la llegada y salidas de los clientes esto se hace para la realización de las actividades y controles de la misma. 


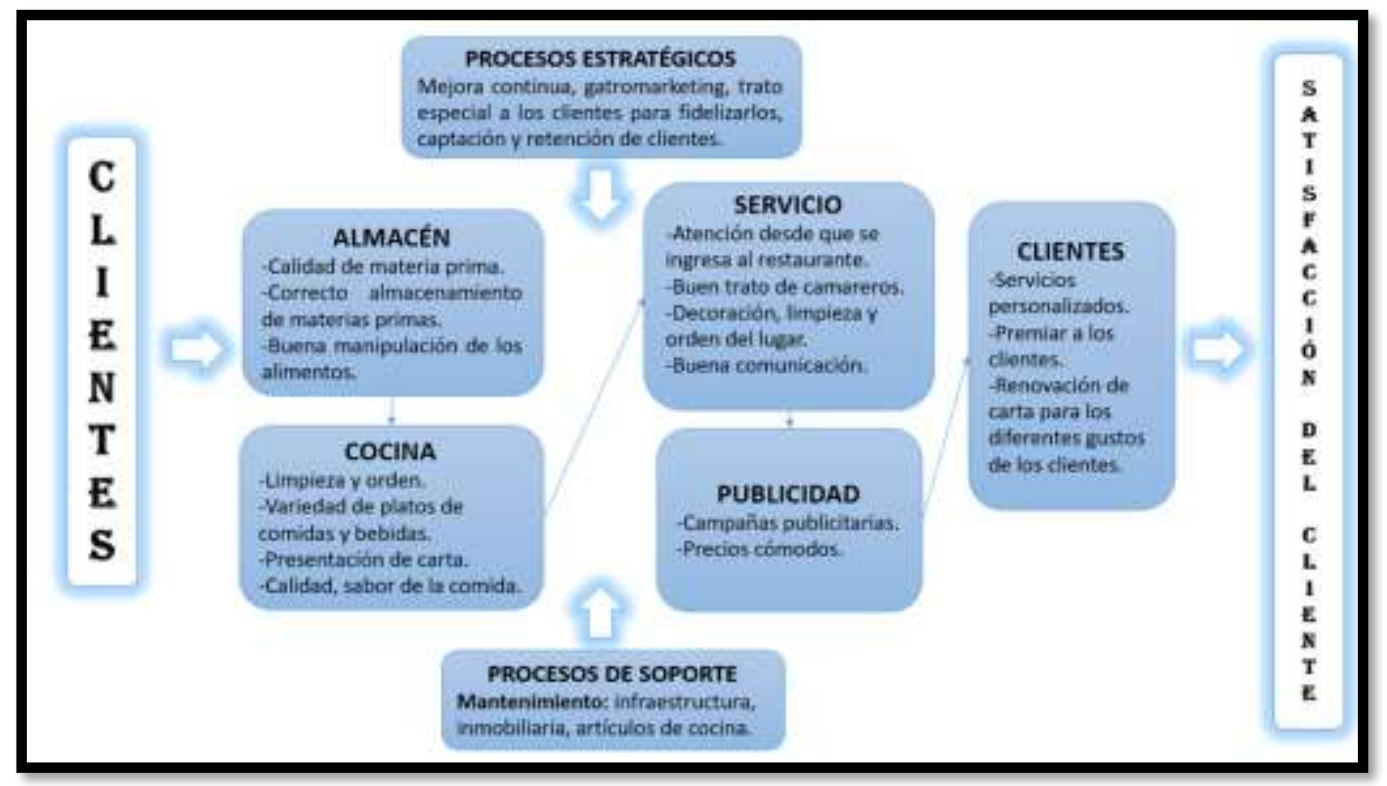

Gráfico 12 Mapa de cada proceso.

\section{Descomposición del propósito de cada proceso en sus objetivos implícitos y evaluación de los mismos.}

El propósito de cada proceso se lo descompone con sus objetivos implícitos para poder realizar el debido control de la mejora continua y el cumplimiento de las mismas. Cada uno de estos objetivos se evalúan mediante la aplicación de los procesos del sistema de calidad.

Para elegir un indicador se debe tener en cuenta, y tiene que ser entendible, controlable por parte del personal que va a desempeñar o llevar a cabo estos procesos.

\section{Cuadro de control de los indicadores.}

Los indicadores se pueden definir como un tipo de instrumentos que permiten medir la consecución o evolución de los objetivos de la empresa basados principalmente en los aspectos clave del negocio u otros aspectos del funcionamiento según el tipo de actividad que realice la empresa. Aunque también se pueden definir como un dato o conjunto de datos que ayudan a medir objetivamente la evolución de un proceso o de una actividad. (Muñiz, 2014)

La forma en que los indicadores pueden informar son:

- Según el tipo de variables sean controladas de forma interna o externamente por la propia empresa.

- La manera que pueden informar debe ser numérica o no, cuantitativa o cualitativa, evolutiva o estática.

- Miden los aspectos clave u otros aspectos en un momento del tiempo y también su evolución. 
El Cuadro de Mando es una herramienta de gestión que facilita la toma de decisiones. Recoge un conjunto coherente de indicadores que proporciona a la alta dirección y al equipo de dirección, una visión comprensible de la evolución de la gestión del conjunto de la organización y de cada área de responsabilidad.

\section{Cuadro para determinar los procesos en los que se debe realizar un plan de calidad.}

Un plan de calidad es información documentada que especifica qué procedimientos de trabajo y recursos se encuentran asociados y se deben aplicar en el proceso, quien son las personas que deben aplicarlos y cuándo tienen que aplicarse a un proyecto, producto, proceso o contrato específico. Los planes de calidad proporcionan una forma de relacionar los requisitos específicos del proceso, producto, proyecto o contrato con los métodos y prácticas de trabajo que apoyan la realización del producto o servicio ofrecido. (ISO 9001, 2018). Para elaborar el cuadro de plan de calidad, primeramente, se construye una tabla con los requisitos del cliente con su definición y los procesos propuestos. Seguidamente esto se valora en un rango de 1 a 5 , considerando que 5 es la máxima puntuación, 3 es la puntuación media y 1 baja puntuación.

\begin{tabular}{|l|c|c|c|}
\hline INDICADORES & & $\mathrm{X}$ & \\
\hline Calidez en la bienvenida & $\mathrm{X}$ & \\
\hline Rapidez para tomar la orden & & $\mathrm{X}$ & \\
\hline Rapidez de preparación & & & $\mathrm{X}$ \\
\hline Sabor y presentación & & & $\mathrm{X}$ \\
\hline Higiene & & & $\mathrm{X}$ \\
\hline Ambiente agradable & & $\mathrm{X}$ & \\
\hline Rapidez en la emisión de factura & & & \\
\hline
\end{tabular}

Gráfico 13: Plan de calidad para Restaurantes de Bahía de Caráquez

\section{Plan de calidad de procesos.}

Un plan de calidad de procesos tiene como prioridad, hacer participar a todos los colaboradores de una institución, e inclusive les dan la potestad de tomar decisiones en consideración a su entendimiento. Entonces a través de esto tenemos que:

- Mantener un Sistema de Gestión de Calidad adecuado a Manufactura y Servicios Mecánicos, que permitan satisfacer las necesidades, expectativas y exigencias de los clientes, basándonos en los requisitos de la Norma ISO 9001.

- Alcanzar los niveles de eficacia en todos los procesos de Sistema de Gestión de Calidad. 
- Mantener la Confiabilidad de operación en $95 \%$

- Fundamentar el Sistema de Gestión de la Calidad en la prevención de no conformidades como un medio que proporcione a los clientes, producto y servicios de calidad.

- Garantizar el cumplimiento de las especificaciones y de los requisitos pautados con el cliente. (JÁCOME, 2013)

\section{Matriz de interrelación de procesos}

Cada uno de los procesos que se rigen dentro de un restaurante y que están relacionadas unos con otros, es necesario realizar una matriz de interrelación de procesos de todos ellos. Se elabora un cuadro donde están los procesos y la relación que existe dando así un orden a seguir dentro de la restauración, sirviendo de orientación a los encargados de cada área.

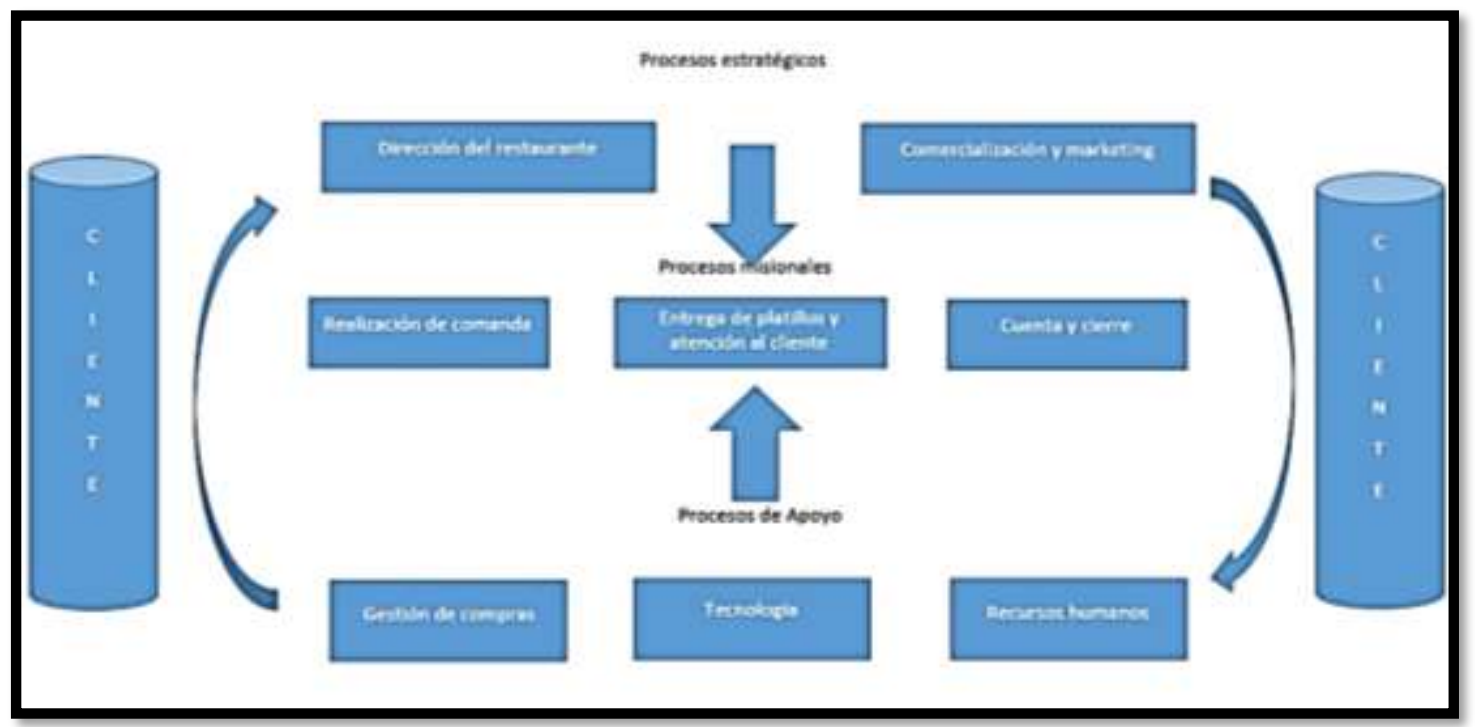

Gráfico 14: Matriz de interrelación de procesos.

\section{Selección, evaluación y reevaluación de proveedores.}

La selección de proveedores es un proceso que está presente en toda organización, debido a que es necesaria la adquisición de bienes y servicios; por lo que este proceso debe ser enfocado a la búsqueda de calidad. En la mayoría de las organizaciones que el departamento de compras o proveeduría es el encargado de realizar la búsqueda y selección de proveedores que mejor se adecuen a las necesidades de la organización. La clave en la selección de los proveedores de un producto o servicio recae en la importancia de saber qué criterios utilizar para seleccionarlos,

\section{Selección de proveedores.}


Involucra una búsqueda exhaustiva de todos los posibles proveedores y se deben eliminar uno a uno conforme a la lista de criterios y diversas consideraciones, hasta reducir a unos pocos a los cuales se les solicitará una cotización.

\section{Auditorías internas.}

Las auditorias en empresas que tienen contacto con alimentos o bebidas juegan un papel fundamental en la garantía de la seguridad de esos alimentos y bebidas. Son una manera de determinar si se están llevando a cabo prácticas de seguridad alimentaria correctas para mantener los estándares de inocuidad de los alimentos que evitan posibles multas e incluso cierres de local tras inspecciones, toxiinfecciones alimentarias que también puedan conducir a multas y cierres y, sobre todo, al mantenimiento de la reputación del negocio. Una auditoría periódica en nuestro restaurante minimizará estos riesgos. (SAIA, 2019)

Según Saia (2019), el objetivo que quiere focalizar las autorías internas es identificar las propiedades organolépticas de determinados productos útiles para nuestro servicio dentro de la industria gastronómica, tales como su sabor, olor, color, textura, apariencia, métodos de conservación y almacenamiento para así minimizar costos y riesgos de infección de virus a las personas.

\section{Conclusiones.}

Todos los establecimientos de alimentos y bebidas dentro del cantón Sucre deben de dar una buena atención al cliente y sobre todo tener en cuenta las medidas de bioseguridad ya que algunos establecimientos se mantienen en stop y no logran acceder a su apertura, sin embargo, algunos establecimientos laboran con poca cantidad de carga.

Dentro de la gestión implica necesariamente que debe mantener a todo su personal capacitado para que así pueda brindar un servicio acorde a las necesidades del cliente por supuesto, porqué ambas partes son importantes dentro del sector turístico.

Con estas acciones de seguridad alimentaria se mejorará la atención al cliente y se reducirá aquellas falencias dentro del establecimiento para el cumplimiento adecuado de las diferentes funciones y medidas de bioseguridad.

La estandarización y formalización de los restaurantes es uno de los puntos más importantes en relación a la mejora del servicio también les permitirá a los establecimientos tener una base para trabajar y sobre la cual desarrollar su mejora continua, además la formalización de los procesos facilita tener una mejor visión de los procesos permitiendo observar las deficiencias de estos y mejorar la eficiencia de estos y de los ejecutores de los mismos.

\section{Bibliografía}

Blogspot. (16 de julio de 2012). ORIGEN DE LOS RESTAURANTES. Obtenido de Blogspot: http://alimentosybebidasute.blogspot.com/2012/07/historia-de-la-restauracion.html 
CuestioPro. (2021). (Cuestión PRO) Obtenido de https://www.questionpro.com/blog/es/formula-paramedir-la-satisfaccion-del-cliente/

Fonseca, E. (2007). Dichos y Refranes. (Lexus, Ed.) Recuperado el 2021, de https://www.dichosyrefranes.net/libro/turismo-hoteleria-y-restaurantes-lexus-pdf.html

ISO 9001. (6 de Marzo de 2018). ¿Cómo se lleva a cabo un plan de calidad para procesos? Obtenido de Nueva ISO 900-2015: https://www.nueva-iso-9001-2015.com/2018/03/plan-de-calidad-procesos/

ISO, T. (s/f). RESTAURANTES / BARES. Obtenido de https://www.isq-turistica.com/sectorestur\%C3\%ADsticos/restaurantes-bares/

JÁCOME, D. C. (Febrero de 2013). “Diseño de un Manual de Sistema de Gestión de Calidad. Obtenido de

UTE: http://repositorio.ute.edu.ec/xmlui/bitstream/handle/123456789/5554/51597_1.pdf?sequence=1\&isAllow $\mathrm{ed}=\mathrm{y}$

Melo,
A.
(2020).

ACADEMIA.

Obtenido

de

https://www.academia.edu/31820428/Historia_de_la_Restauraci\%C3\%B3n

MESTRES, M. (08 de Abril de 2019). Gestión basada en procesos . Obtenido de Captio: https://www.captio.net/blog/identificar-y-elaborar-el-mapa-de-procesos-de-laempresa\#: :text=El\%20mapa\%20de $\% 20$ procesos $\% 20$ es,diferentes $\% 20$ departamentos $\% 20 \mathrm{de} \% 201 \mathrm{a} \% 20 \mathrm{e}$ mpresa.

Muñiz, L. (16 de Mayo de 2014). El Cuadro de Mando: soporte del. Obtenido de ACCID: https://accid.org/wpcontent/uploads/2018/10/DISENO_DE_CUADROS_DE_MANDO_INDICADORES_ACCID.pdf OCAÑA, T. P. (2018). DISEÑO DE UN SISTEMA DE GESTIÓN DE LA CALIDAD CONFORME A LA ISO. Obtenido de ESPOCH: http://dspace.espoch.edu.ec/bitstream/123456789/8284/1/42T00441.pdf Qualite. (25 de julio de 2019). ISO 22001: Beneficios de la Norma de Inocuidad Alimenticia. Obtenido de Qualite: http://www.qualite.mx/noticias/iso-22001-beneficios-de-la-norma-de-inocuidad-alimenticia Riquelme, M. (03 de Septiembre de 2017). Costos de Calidad ¿Qué son y en que consisten? Obtenido de Web y $\quad$ Empresas : https://www.webyempresas.com/costos-de- 
calidad/\#: :text=\%C2\%BFQu\%C3\%A9\%20son\%20costos\%20de\%20calidad,para\%20alcanzar\%20par\% C3\%A1metros\%20de\%20excelencia.

Riquelme, M. (05 de Febrero de 2019). Organigrama de un Restaurante. Obtenido de webyempresas: https://www.webyempresas.com/organigrama-de-unrestaurante/\#: :text=El\%20organigrama\%20de\%20un\%20restaurante,le\%20dan\%20vida\%20al\%20mism o.

SAIA. (07 de Febrero de 2019). Auditoría en un restaurante: en qué consiste y cómo prepararlas. Obtenido de Saia.es: https://saia.es/auditoria-en-un-restaurante-en-que-consiste-y-comoprepararla/\#: :text=\%C2\%BFEn\%20qu\%C3\%A9\%20consiste\%20una\%20auditor\%C3\%ADa,ha\%20mar cado\%20con\%20la\%20auditor\%C3\%ADa

Sanchez, J. C., Avalos, R. B., \& Vinueza, J. A. (04 de Marzo de 2019). Un estudio de la calidad del servicios en Restaurantes en Ecuador. Obtenido de Revista Espacios: http://www.revistaespacios.com/a19v40n07/a19v40n07p09.pdf

Sepúlveda, M. S. (14 de Diciembre de 2013). Escuela de organización industrial . Obtenido de https://www.eoi.es/blogs/mintecon/2013/12/14/tecnica-o-herramienta-util-en-la-direccion-de-proyectosfoda/\#: :text=El\%20an\%C3\%A1lisis\%20FODA\%20es\%20una,los\%20objetivos\%20y\%20pol\%C3\%AD ticas\%20formulados.

Serrano, S. C. (Noviembre de 2017). CLASIFICACIÓN DE LOS COSTES DE CALIDAD EN LA GESTIÓN. Obtenido de Universidad de Valencia: https://www.uv.es/scliment/investigacion/2005/partdoblenov2005.prn.pdf

TURISTICAS, I. (20 de junio de 2020). RESTAURANTES / BARES. Obtenido de ISQ TURISTICAS: https://www.isq-turistica.com/sectores-tur\%C3\%ADsticos/restaurantes-bares/ 